\title{
Programmed death-ligand 1 expression level as a predictor of EGFR tyrosine kinase inhibitor efficacy in lung adenocarcinoma
}

\author{
Minsu Kang ${ }^{1 \#}$, Changhee Park ${ }^{2 \#}$, Se Hyun Kim ${ }^{1}$, Sock Won Yoon ${ }^{1}$, Koung Jin Suh ${ }^{1}$, Yu Jung Kim ${ }^{1}$, \\ Chan-Young Ock ${ }^{2}$, Miso Kim", Bhumsuk Keam², Tae Min Kim², Dong-Wan Kim², Dae Seog Heo ${ }^{2}$, \\ Jong Seok Lee ${ }^{1}$ \\ ${ }^{1}$ Department of Internal Medicine, Seoul National University Bundang Hospital, Seoul National University College of Medicine, Seongnam, Korea; \\ ${ }^{2}$ Department of Internal Medicine, Seoul National University Hospital, Seoul National University College of Medicine, Seoul, Korea \\ Contributions: (I) Conception and design: SH Kim; (II) Administrative support: SH Kim, B Keam, SW Yoon; (III) Provision of study materials or \\ patients: M Kang, C Park; (IV) Collection and assembly of data: All authors; (V) Data analysis and interpretation: SH Kim, CY Ock, M Kang, C \\ Park; (VI) Manuscript writing: All authors; (VII) Final approval of manuscript: All authors. \\ \#These authors contributed equally to this work. \\ Correspondence to: Se Hyun Kim, MD, PhD. Division of Hematology-Oncology, Department of Internal Medicine, Seoul National University \\ Bundang Hospital, 82, Gumi-ro, Bundang-gu, Seongnam, 13620, Republic of Korea. Email: sehyunkim@snubh.org.
}

Background: The main objective of this study was to investigate the impact of programmed death-ligand 1 (PD-L1) expression on the efficacy of epidermal growth factor receptor (EGFR) tyrosine kinase inhibitors (TKI) in patients with advanced non-small cell lung cancer (NSCLC).

Methods: This study analyzed 108 patients with NSCLC who had received EGFR-TKI as first-line systemic treatment at Seoul National University Bundang Hospital and Seoul National University Hospital between December 2012 and October 2018. The National Cancer Center Research Institute (NCCRI) and The Cancer Genome Atlas (TCGA) datasets were analyzed to investigate the mechanisms underlying EGFR-TKI-resistance in tumors with high PD-L1 expression.

Results: Among the 108 patients, 55, 37, and 16 had negative (PD-L1 Tumor proportion score <1\%), weak (1-49\%), and strong ( $\geq 50 \%$ ) PD-L1 expression, respectively. Patients with strong PD-L1 expression had significantly shorter median progression-free survival (PFS; 7.07 months) than patients with weak (14.73 months, $\mathrm{P}<0.001)$ or negative $(12.70$ months, $\mathrm{P}=0.001)$ PD-L1 expression. After adjustment for covariates by Cox regression, PD-L1 expression remained a significant indicator of adverse prognosis. In EGFR-TKI-refractory patients, the frequency of T790M mutation and the PFS following treatment with third-generation EGFR-TKI and PD-1 antibody were similar in the three groups. TCGA and NCCRI database analysis showed that high PD-L1 expression in EGFR-mutated NSCLCs correlated with IL-6/JAK/STAT3 signaling and high MUC16 mutation frequency.

Conclusions: Strong PD-L1 expression in tumors might be a surrogate indicator of poor response to firstline EGFR-TKIs in NSCLC patients with sensitizing EGFR mutations, and may reflect a de novo resistance mechanism involving JAK-STAT signaling.

Keywords: Non-small cell lung cancer (NSCLC); epidermal growth factor receptor (EGFR); programmed deathligand 1 (PD-L1); EGFR tyrosine kinase inhibitor (EGFR TKI); JAK-STAT pathway

Submitted Aug 01, 2020. Accepted for publication Dec 13, 2020.

doi: $10.21037 /$ tlcr-20-893

View this article at: http://dx.doi.org/10.21037/tlcr-20-893 


\section{Introduction}

In patients with advanced non-small cell lung cancer (NSCLC) whose tumors harbor activating epidermal growth factor receptor $(E G F R)$ mutations (exon 19 deletions or L858R point mutations), treatment with EGFR tyrosine kinase inhibitors (TKIs) is recommended (1-4). Patients with EGFR-mutated advanced NSCLC initially respond to first-generation EGFR-TKIs, such as gefitinib or erlotinib, but the majority of them usually show disease progression within one year after treatment initiation (5). The reason for acquired drug resistance is the development of a second mutation in exon 20 of the EGFR gene, causing a threonine (T)-to-methionine (M) substitution at codon 790 (T790M). This mutation is observed in $50-60 \%$ of the patients with acquired resistance to first-generation EGFR-TKIs (6). Although a second-generation EGFR-TKI, afatinib, has been developed to solve this issue, various clinical studies showed its limited efficacy against lung cancers resistant to first-generation EGFR-TKIs $(7,8)$. Subsequently, a thirdgeneration EGFR-TKI, osimertinib, has been approved for the treatment of T790M-positive lung tumors with acquired resistance to gefitinib, erlotinib or afatinib (9). More recently, the clinical practice guidelines of the National Comprehensive Cancer Network recommended osimertinib as the preferred EGFR-TKI option for firstline treatment (2).

Recent studies of cancer immunology have brought about a novel therapeutic concept in oncology. Tumor cells may escape the immune system by expressing programmed death-1 ligand (PD-L1) on their surface. The binding of tumor cell PD-L1 to programmed death-1 (PD-1) on the surface of activated $\mathrm{T}$ cells downregulates the immune response reducing the production and cytotoxic activity of inflammatory cytokines (10). This mechanism of immune evasion is supported by the following observations: (I) in a substantial proportion of NSCLC patients, PDL1 expression in the tumor increases after platinumbased neoadjuvant chemotherapy, and this upregulation is associated with poor clinical outcome (11); (II) NSCLC patients with survival time shorter than 3 years after lobectomy surgery show relatively high expression of PDL1 (12). Immune checkpoint inhibitors targeting the PD-1/ PD-L1 axis represent a new paradigm for the treatment of advanced NSCLC. Recent studies have shown that PD-1/ PD-L1 blockade increases the number of effector T cells, as well as the cytotoxic activity of tumor-specific T cells (13). Immune checkpoint inhibitors targeting the PD-1/PD-L1 axis revert the immune inhibitory effect of tumors, allowing immune cells to kill cancer cells. Notably, in patients with advanced NSCLC treated with immune checkpoint inhibitors, PD-L1 expression in tumor cells is a positive predictor of survival (14).

Although most patients with advanced NSCLC carrying EGFR mutations show a durable response to EGFRTKIs, some of them undergo rapid disease progression or primary drug resistance. Specific features are associated with a relatively high probability to benefit from EGFRTKIs: Asian origin, female gender, adenocarcinoma histology, and non-smoking status (15). However, the relationship between tumor cell-specific PD-L1 expression and the clinical outcome of advanced NSCLC patients treated with EGFR-TKI is unclear. We here investigated whether PD-L1 expression in tumor cells could be used to predict the duration of treatment, as well as the emergence of the T790M mutation, in patients with advanced NSCLC receiving EGFR-TKI as a first-line systemic treatment. Publicly available transcriptome datasets of lung adenocarcinoma were analyzed to interpret the findings of this study. We present the following article in accordance with the REMARK reporting checklist (available at http:// dx.doi.org/10.21037/tlcr-20-893).

\section{Methods}

\section{Patients}

This study analyzed 108 patients with advanced NSCLC who had received EGFR-TKI as a first-line systemic treatment at Seoul National University Bundang Hospital and Seoul National University Hospital between December 2012 and October 2018 (Figure 1). Demographic characteristics and clinical data, including age, gender, smoking status, Eastern Cooperative Oncology Group Performance Status (ECOG PS), baseline EGFR mutation status, type of EGFR-TKI treatment, progression-free survival (PFS), and patient response, based on the Response Evaluation Criteria in Solid Tumors (RECIST, Version 1.1), were extracted from retrospective electronic medical records. The rate of the T790M mutation following failure of EGFR-TKI treatment, as well as PFS and patient response to third-generation EGFR-TKIs and subsequent immunotherapy, were also reviewed. This study was performed in accordance with the Declaration of Helsinki (as revised in 2013). This study was approved by the Institutional Review Board of Seoul National University 


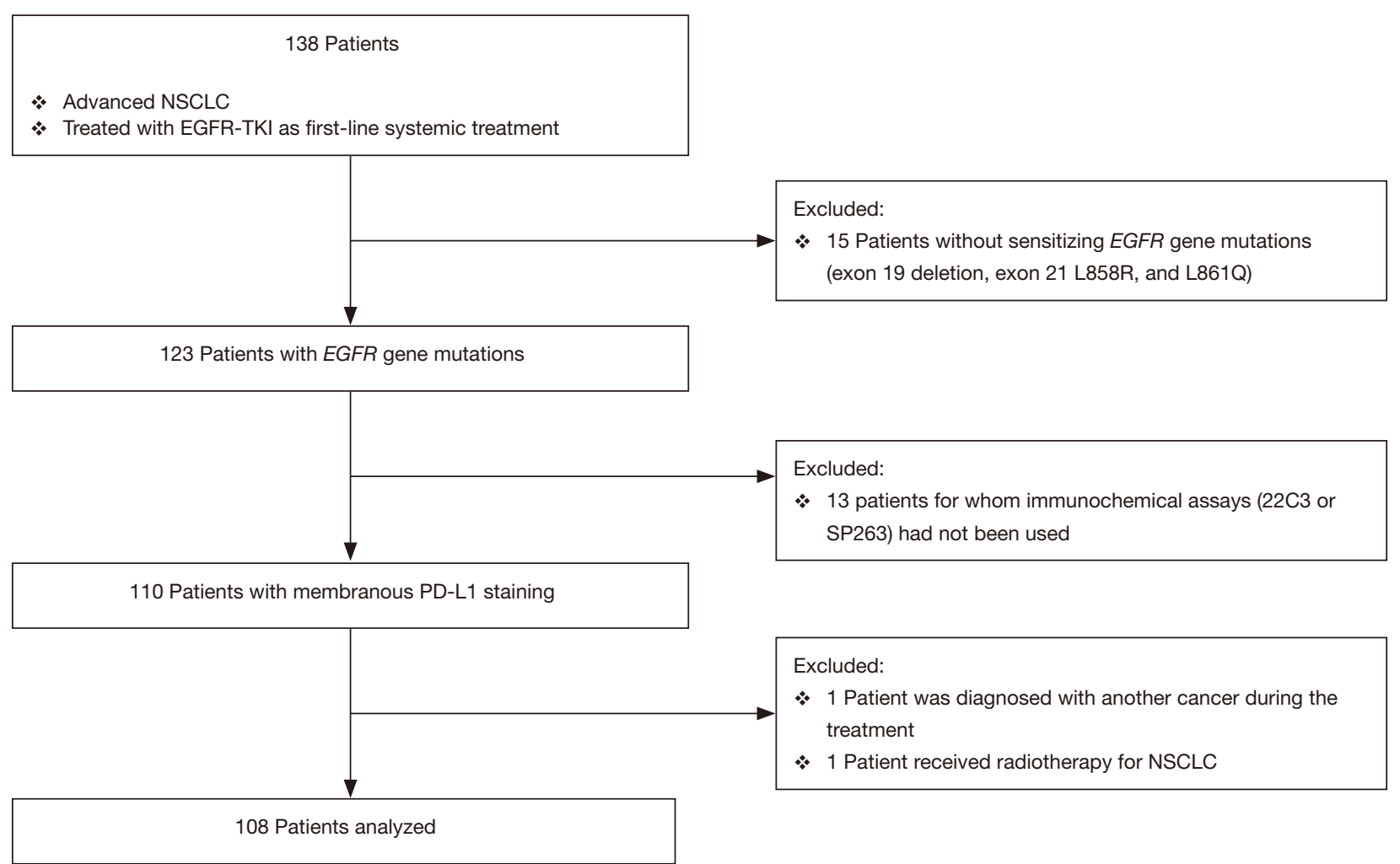

Figure 1 CONSORT diagram.

Bundang Hospital (IRB No. B-1808/487-101). Consent to participate was waived by the ethics board.

\section{Analysis of EGFR mutations and PD-L1 expression}

EGFR gene mutations were analyzed by a peptide nucleic acid (PNA) clamping-based sensitive method (PNA Clamp $^{\mathrm{TM}}$ or PANAMutyper ${ }^{\mathrm{TM}}$ EGFR mutation detection kit) in formalin-fixed paraffin-embedded tissues (16-18). $E G F R$-activating mutations were defined as mutations associated with EGFR-TKI sensitivity, including exon 19 deletion, exon 21 L858R, and L861Q.

Diagnostic assays based on PD-L1 immunohistochemistry were performed according to the manufacturer's instructions. The following antibodies and detection systems were utilized: mouse monoclonal primary anti-PD-L1 antibody, 22C3 pharmDx (prediluted, clone 22C3, Dako, Carpinteria, CA, USA) with an Autostainer Link 48 and an EnVision DAB Detection System (Agilent Technologies, Santa Clara, CA, USA); rabbit monoclonal primary anti-PD-L1 antibody, Ventana SP263 (prediluted, Ventana Medical Systems, Tucson, AZ, USA) with a Benchmark XT staining system and
Ultra with OptiView Universal DAB Detection Kit (Ventana Medical Systems). The staining results were interpreted by expert lung pathologists. The percentage of tumor cells with membranous PD-L1 staining of any intensity (tumor proportion score, TPS) was categorized into three ranges and the corresponding groups $(<1 \%$, negative expression; $1-49 \%$, weak expression; $\geq 50 \%$, strong expression).

\section{Analysis of open source databases of RNA expression}

To evaluate the impact of PD-L1 expression on the efficacy of EGFR-TKI treatment in patients with lung adenocarcinoma carrying EGFR mutations, we used two publicly available transcriptome datasets of lung adenocarcinoma: the National Cancer Center Research Institute (NCCRI) dataset and The Cancer Genome Atlas (TCGA) dataset $(19,20)$. The NCCRI dataset was downloaded from Gene Expression Omnibus (https://www. ncbi.nlm.nih.gov/geo/, accession number GSE31210), and the EGFR mutational status of patients in the NCCRI dataset was available from the literature. The TCGA transcriptome dataset and curated mutation data were 
Table 1 Baseline characteristics of patients

\begin{tabular}{lc}
\hline Characteristic & Number of patients (\%) \\
\hline Age & 65 \\
Median & $34-84$ \\
Range & \\
Sex, n (\%) & $71(65.7)$ \\
Female & $37(34.3)$ \\
Male & \\
Smoking history, n (\%) & $73(67.6)$ \\
Never smoker & $35(32.4)$ \\
Current/former smoker & \\
ECOG PS, $\mathrm{n}$ (\%) & $96(88.9)$ \\
$<2$ & $12(11.1)$ \\
$\geq 2$ & \\
Brain metastases at diagnosis, $\mathrm{n}(\%)$ & $60(55.6)$ \\
No & $45(44.4)$ \\
Yes & $63(58.3)$ \\
EGFR mutation, $\mathrm{n}(\%)$ & \\
Exon 19 deletion & \\
\hline
\end{tabular}

ECOG PS, Eastern Cooperative Oncology Group Performance Status; EGFR, epidermal growth factor receptor.

downloaded from the cBioPortal (https://www.cbioportal. org/) (21). Expression values were log2-normalized to genes using Cluster 3.0 software (22).

The PD-L1 status of samples was classified according to the level of CD274 (PD-L1) mRNA expression; samples with upper $50 \%$ values throughout the whole samples were defined as PD-L1-high, and the remaining lower 50\% values were defined as PD-L1-low (23). Then, synonymous and benign mutations were excluded, and samples with potentially pathogenic EGFR mutations were selected. Only samples for which transcriptomic data were available and the EGFR mutational status was known were included in the analysis.

To compare pathway enrichment in PD-L1-high and PD-L1-low samples, Gene Set Enrichment Analysis was performed (GSEA_4.0.2, http://software.broadinstitute. org/gsea/index.jsp) (24,25). Hallmark gene sets from the Molecular Signatures Database (MsigDB) were analyzed (26). In the GSEA analysis, pathways with nominal
$\mathrm{P}$ value $<0.05$ and FDR q value $<0.25$ were considered as significantly enriched.

\section{Statistical analysis}

The correlation between tumor PD-L1 expression and other patient characteristics was analyzed by either chi-square test or Fisher's exact test. PFS was defined as the time from the initiation of EGFR-TKI treatment to the detection of disease progression or any cause of death, and was evaluated with the Kaplan-Meier method. The log-rank test was applied to compare the cumulative survival time between patient groups. Multivariate analysis was performed using a logistic regression model incorporating age, sex, smoking history, ECOG PS, status of brain metastasis at diagnosis, and PD-L1 expression. The mutational profiles of PD-L1high and PD-L1-low patients were compared by using the TCGA dataset. To establish whether a certain gene was more frequently mutated in a specific group, Fisher's exact test was applied. All statistical analyses were performed with $\mathrm{R}$ software version 3.6.0 (https://www.r-project.org/), and $\mathrm{P}$ values $<0.05$ were considered indicative of statistical significance.

\section{Results}

Outcome of patients treated with EGFR-TKI as a first-line systemic therapy

A total of 108 patients were included in the analysis; the demographic and clinical characteristics of participants are shown in Table 1. The median age was 65 years (range, 34-84 years), 71 (65.7\%) patients were female, 73 (67.6\%) never-smokers, 96 (88.9\%) displayed ECOG PS <2, and $45(41.7 \%)$ had no brain metastases when diagnosed. In addition, $60(55.6 \%)$ patients presented exon 19 deletions and $48(44.4 \%)$ exon 21 deletions. All patients had been treated with EGFR-TKI as appropriate.

Among the 108 lung adenocarcinoma specimens analyzed, $55(50.9 \%), 37$ (34.3\%), and $16(14.8 \%)$ exhibited negative (TPS $<1 \%)$, weak (1-49\%), and strong $(\geq 50 \%)$ PD-L1 expression, respectively. PD-L1 expression was not correlated with age, sex, smoking history, ECOG PS, presence of brain metastasis at diagnosis, type of EGFR mutation, or type of EGFR-TKI used (Table 2).

In univariate analysis, the median PFS of EGFRTKI-treated patients was 12.70 months (95\% CI, 9.66-15.74 months) for patients with negative PD-L1 
Table 2 Association between PD-L1 expression and clinicopathologic features

\begin{tabular}{|c|c|c|c|c|}
\hline PD-L1 expression & Negative $(n=55,100 \%)$ & Weak $(n=37,100 \%)$ & Strong $(n=16,100 \%)$ & $P$ value \\
\hline$<65$ & $29(52.7)$ & $14(37.8)$ & $9(56.25)$ & \\
\hline$\geq 65$ & $26(47.3)$ & $23(62.2)$ & $7(43.75)$ & \\
\hline Sex & & & & 0.331 \\
\hline Male & $17(30.9)$ & $16(43.2)$ & $4(25.0)$ & \\
\hline Smoking history & & & & 0.164 \\
\hline Never smoker & $39(70.9)$ & $21(56.8)$ & $13(81.3)$ & \\
\hline Current/former smoker & $16(29.1)$ & $16(43.2)$ & $3(18.8)$ & \\
\hline$\geq 2$ & $4(7.3)$ & 7 (18.9) & $1(6.3)$ & \\
\hline Brain metastases at diagnosis & & & & 0.764 \\
\hline No & $22(40.0)$ & $15(40.5)$ & $8(50.0)$ & \\
\hline Yes & $33(60.0)$ & $22(59.5)$ & $8(50.0)$ & \\
\hline Oncogenic driver & & & & 0.560 \\
\hline Exon 19 del & $33(60.0)$ & $18(48.6)$ & $9(56.3)$ & \\
\hline Exon $21 \mathrm{~L} 858 \mathrm{R}$ & $22(40.0)$ & $19(51.4)$ & $7(43.8)$ & \\
\hline Partial response & $42(76.4)$ & $29(78.4)$ & $13(81.3)$ & \\
\hline Stable disease & $7(12.7)$ & $7(18.9)$ & $0(0.0)$ & \\
\hline Progressive disease & $6(10.9)$ & $1(2.7)$ & $3(18.8)$ & \\
\hline 2nd Mutation & & & & 0.838 \\
\hline T790M & $21(38.2)$ & $13(35.1)$ & $7(43.8)$ & \\
\hline Subsequent treatment & & & & $<0.001$ \\
\hline Immunotherapy & $7(12.7)$ & $2(5.4)$ & $9(56.3)$ & \\
\hline \multicolumn{5}{|l|}{ Response rate $^{a}$} \\
\hline EGFR-TKIs & $42 / 55(76.4)$ & 29/37 (78.4) & $13 / 16(81.3)$ & 1.000 \\
\hline Third generation EGFR-TKI & 6/23 (26.1) & $3 / 10(30.0)$ & $4 / 7(57.1)$ & 0.335 \\
\hline Immune checkpoint inhibitors & 0/6 (0.0) & $0 / 2(0.0)$ & $1 / 7(14.3)$ & 1.000 \\
\hline
\end{tabular}

a , number of patients with partial response/total number of patients. PD-L1, programmed death-ligand 1; ECOG PS, eastern cooperative oncology group performance status; EGFR, epidermal growth factor receptor; TKI, tyrosine kinase inhibitor. 


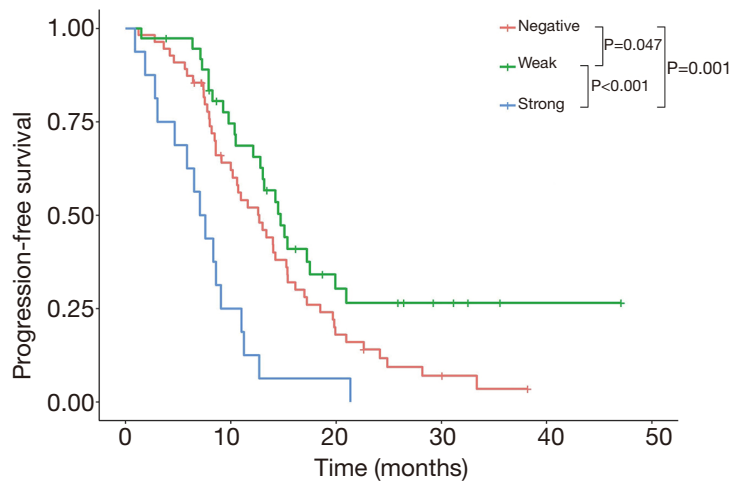

Figure 2 Kaplan-Meier curve of progression-free survival in patients receiving first-line EGFR-TKI therapy. EGFR, epidermal growth factor receptor; TKI, tyrosine kinase inhibitor.

expression, 14.73 months (95\% CI, 12.13-17.33 months) for patients with weak PD-L1 expression, and 7.07 months (95\% CI, 4.98-9.16 months) for patients with strong PDL1 expression (log rank; negative vs. weak PD-L1 expression, $\mathrm{P}=0.047$; negative vs. strong $\mathrm{PD}-\mathrm{L} 1$ expression, $\mathrm{P}=0.001$; weak vs. strong PD-L1 expression, $\mathrm{P}<0.001$ ) (Figure 2). Patients with strong PD-L1 expression had a significantly shorter PFS compared to those with weak or negative PD-L1 expression.

When adjusted for age, smoking history, sex, ECOG PS, and status of brain metastasis at diagnosis in the Cox regression model, PD-L1 expression remained a significant prognostic indicator of short-term PFS (hazard ratio for negative vs. strong PD-L1 expression, 2.788; 95\% CI, 1.536-5.059; $\mathrm{P}=0.001$ ) (Table 3).

Early progression (within 6 months after first-line EGFR-TKI treatment) rates were $12.7 \%, 2.7 \%$, and $37.5 \%$ in patients with negative, weak, and strong PD-L1 expression respectively (Fisher's exact test; negative $v s$. weak $v s$ s strong PD-L1 expression, $\mathrm{P}=0.004)$. Notably, most cases of early progression were observed among patients with high PD-L1 expression (Figure 3).

There were no statistically significant PD-L1 expressionrelated differences in the best treatment response of EGFRTKI, based on RECIST 1.1 criteria (negative vs. weak vs. strong PD-L1 expression, $\mathrm{P}=0.133$ ) (Table 2). However, the disease control rate (partial response + stable disease) was numerically lower in strong PD-L1 expression group (81.3\%) compared to those of negative (89.1\%) and weak (97.3\%) group.

\section{Acquired resistance to first-line EGFR TKI treatment}

Of 108 patients with advanced NSCLC who had received with EGFR-TKI as first-line systemic treatment, biopsies were performed again in 66 patients after showing acquired resistance. The rate of T790M mutation was analyzed to identify possible differences related to PDL1 expression. Among EGFR-TKI-refractory patients, the rate of T790M mutation was between the three PDL1 expression groups (38.2\% in negative, $35.1 \%$ in weak, and $43.8 \%$ in strong PD-L1 expression group; $\mathrm{P}=0.838$ ). The rate of T790M mutation was associated with neither subtype of EGFR mutation or pretreated EGFR-TKIs (Tables S1,S2).

Among patients treated with third-generation EGFRTKI $(n=47)$, the median PFS was $6.63,8.13$, and 9.77 months for negative, weak and strong PD-L1 expression group respectively. However, these differences were not statistically significant (log rank test: negative $v s$. weak PD-L1 expression, $\mathrm{P}=0.987$; negative $v$ s. strong PD$\mathrm{L} 1$ expression, $\mathrm{P}=0.710$; weak vs. strong $\mathrm{PD}-\mathrm{L} 1$ expression, $\mathrm{P}=0.516$ ) (Figure $\mathrm{S} 1$ ). Moreover, no statistically significant differences related to PD-L1 expression were detected in the response to third-generation EGFR-TKI, based on RECIST 1.1 criteria (negative $v s$. weak $v s$. strong PD-L1 expression, $\mathrm{P}=0.530$ ).

\section{Outcome of patients treated with immune checkpoint inbibitors following EGFR-TKI failure}

After EGFR-TKI treatment failure, 18 patients were treated with anti-PD-1 antibody (Nivolumab; $\mathrm{n}=11$, Pembrolizumab; $\mathrm{n}=7$ ). The median PFS after immune checkpoint inhibition was 1.33 months in negative, 0.43 months in weak, and 1.53 months in strong PDL1 expression group (log rank; negative $v s$. weak PD-L1 expression, $\mathrm{P}=0.326$; negative $v$ s. strong $\mathrm{PD}-\mathrm{L} 1$ expression, $\mathrm{P}=0.559$; weak vs. strong $\mathrm{PD}-\mathrm{L} 1$ expression, $\mathrm{P}=0.138$ ) (Figure S2). At six months, the PFS rate was highest in patients with strong PD-L1 expression (negative PD-L1 expression, $16.7 \%$; weak PD-L1 expression, $0 \%$; strong PD-L1 expression, 41.7\%). However, based on RECIST (Version 1.1), the level of PD-L1 expression did not significantly affect the response to immune checkpoint inhibitors $(\mathrm{P}=0.736)$.

\section{Use of public databases to explore the mechanism of EGFR- TKI resistance}

A total of 127 (50 PD-L1-high) and 64 (25 PD-L1-high) samples of lung adenocarcinoma carrying the EGFR 
Table 3 Multivariate analysis of clinicopathological features for progression-free survival

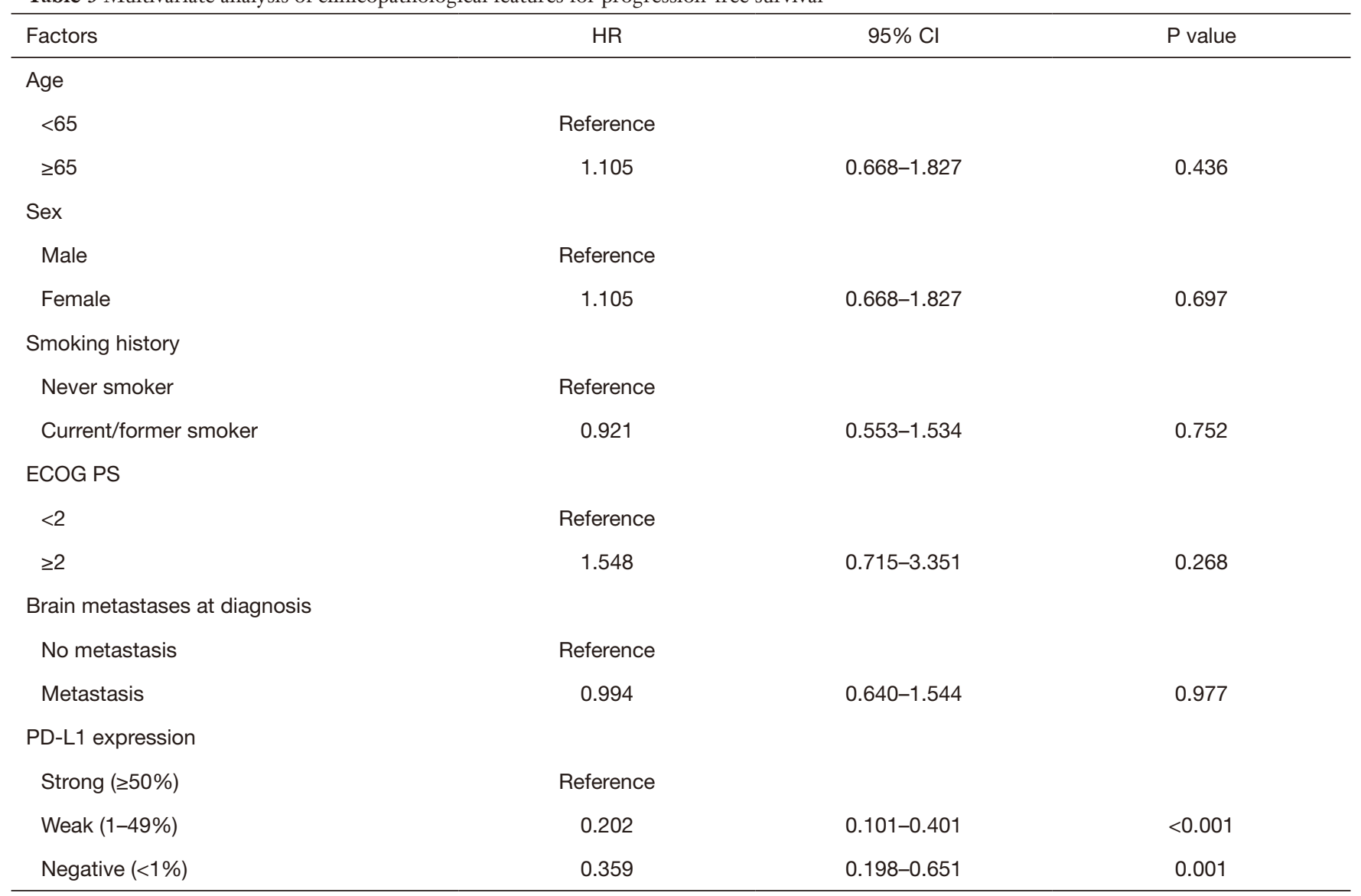

ECOG PS, Eastern Cooperative Oncology Group Performance Status; PD-L1, programmed death-ligand 1.

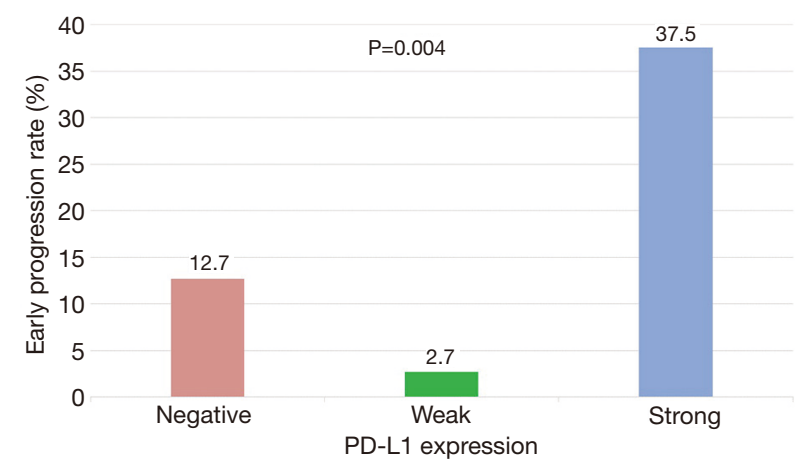

Figure 3 Early progression rate according to PD-L1 expression. PD-L1, programmed death-ligand 1.

mutation were analyzed in the NCCRI and TCGA dataset, respectively. GSEA analysis revealed a significant enrichment of 14 (Table S3) and 8 (Table S4) pathways in PD-L1-high tumors, according to the NCCRI and the
TCGA dataset, respectively. Among these pathways, 8 were significantly enriched in both datasets and most of them were related to immune functions (Figure $4 A, B$ ). Notably, the "HALLMARK_IL6_JAK_STAT3_SIGNALING" pathway, which is known to be associated with EGFRTKI resistance, was consistently and highly enriched in both datasets (normalized enrichment scores of 1.73 and 1.69 in the NCCRI and TCGA dataset, respectively, Figure $4 C, D)(27,28)$.

The comparison of mutational profiles in PD-L1-high and PD-L1-low tumors allowed to identify 26 genes with significantly higher mutation rate in one of the two groups (Table S5). Notably, PD-L1-high samples exhibited a higher frequency of the MUC16 mutation compared to the other samples $(44.0 \%$ vs. $30.8 \%, \mathrm{P}=0.034$, Figure $4 E)$, which is involved in tumorigenesis and metastasis of lung cancer cells through the regulation of the JAK2-STAT3 axis (29). 

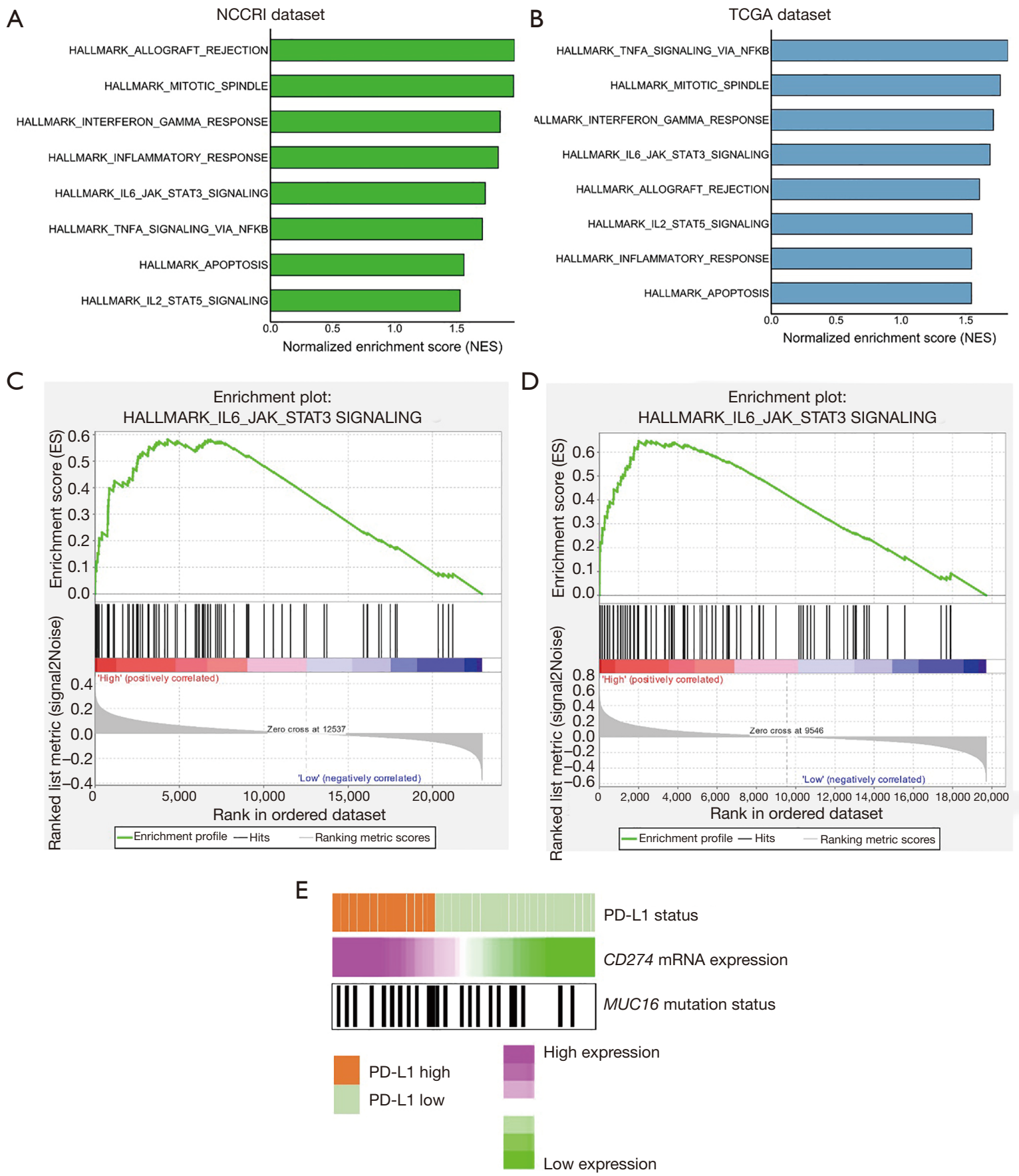

Figure 4 Open-source database analysis focusing on the mechanism of EGFR-TKI resistance in PD-L1-high lung adenocarcinoma. (A) Bar plot showing normalized enrichment score in the NCCRI dataset. The 8 pathways enriched in both the NCCRI and TCGA datasets are shown. (B) Bar plot showing the normalized enrichment score in the TCGA dataset. The 8 enriched pathways common to both datasets are shown. (C) GSEA enrichment plot of the "HALLMARK_IL6_JAK_STAT3_SIGNALING" pathway in the NCCRI dataset. (D) GSEA enrichment plot of the "HALLMARK_IL6_JAK_STAT3_SIGNALING" pathway in the TCGA dataset. (E) Graph showing the status of PD-L1 expression and MUC16 mutation in each sample. Each column represents one sample; the columns are arranged in order of decreasing CD274 (PD-L1) mRNA expression. EGFR, epidermal growth factor receptor; TKI, tyrosine kinase inhibitor; PDL1, programmed death-ligand 1; NCCRI, the national cancer center research institute; TCGA, the cancer genome atlas; GSEA, gene set enrichment analysis; JAK, Janus kinase; STAT, signal transducer and activator of transcription; mRNA, messenger ribonucleic acid. 


\section{Discussion}

In this study, we demonstrated that high PD-L1 expression was associated with poor clinical outcome in patients treated with first-line EGFR-TKI. Early cancer progressors with high PD-L1 expression didn't have significantly more frequent T790M resistance mutation compared to those with low PD-L1 expression.

To explore the mechanism underlying the different efficacy of EGFR-TKI in patients with PD-L1-high and PD-L1-low, EGFR-mutated lung adenocarcinoma, public datasets were employed. A comprehensive analysis of TCGA and NCCRI databases consistently showed that high PD-L1 expression in EGFR-mutated lung adenocarcinoma was correlated with the activation of the IL-6/JAK/STAT3 pathway, as well as with a high frequency of MUC16 mutation.

The association between high PD-L1 expression and de novo resistance to EGFR-TKI of lung adenocarcinoma has been described by previous studies (30-32). The proportion of PD-L1 positive patients ranged between $45.5 \%$ and $66.7 \%$ among those with primary resistance to EGFR-TKI, and between $12.3 \%$ and $30.2 \%$ among those with disease control or acquired resistance $(30,31)$. In addition, it was reported that, after treatment with EGFR-TKI, patients with PD-L1 expression $\geq 50 \%$ exhibited median PFS of 1.6, 3.8, and 5.9 months, while median PFS of 7.3, 9.5, and 12.5 months were observed in patients with PD-L1 expression $<1 \%$, respectively (30-32), which was consistent with our findings. More recently, FLAURA trial (33) reported that, after first-line treatment with gefitinib or erlotinib, median PFS was 6.9 months in patients with PDL1 expression $\geq 1 \%$, while median PFS was 10.9 months in patients with PD-L1 expression $<1 \%$, which was consistent with our results. However, interestingly, after first-line treatment with osimertinib, median PFS was unaffected by PD-L1 expression status.

One of the above-mentioned studies demonstrated the efficacy of immune checkpoint inhibitors in the treatment of EGFR-mutated PD-L1 high tumors, as these tumors are highly positive to CD8+ T cells $(30,34)$; moreover, one of these patients showed PFS longer than 5 months following treatment with an immune checkpoint inhibitor (30). Although the level of PD-L1 expression did not seem to significantly affect PFS in EGFR-TKIresistant patients that had been treated with immune checkpoint inhibitor, some patients with high tumor expression of PD-L1 did show more favorable outcomes, with PFS >6 months, suggesting that subsets of PD-L1high patients might benefit from treatment with immune checkpoint inhibitors. Therefore, although immunotherapy showed limited overall efficacy against EGFR-mutated lung adenocarcinoma $(35,36)$, the specific effects of the immune check point inhibitors in patients with high PD-L1 expression may deserve further investigation.

In addition, immune checkpoint inhibitor in combination with platinum-based chemotherapy may be a possible therapeutic option in EGFR-TKI-resistant patients as the recent IMpower 150 trial reported that chemo-immunotherapy plus bevacizumab improved PFS when compared to chemotherapy alone in EGFR-mutant patients who had progressed on a EGFR-TKI therapy (37). The IMpower 130 trial failed to demonstrate a clinical benefit for the addition of immune checkpoint inhibitor to chemotherapy without bevacizumab in EGFR-mutant NSCLC (38). Clinical benefit between immune checkpoint inhibitor alone and in combination with chemotherapy in EGFR-TKI-resistant patients is not yet studied well.

We showed that the rate of T790M mutation in EGFRTKI-resistant patients did not significantly differ between groups with different levels of tumor PD-L1 expression. This finding contrasts with a previous report (32), showing a higher frequency of the mutation in PD-L1-negative compared to PD-L1-high patients. This contradiction could be due to differences in the number of patients who underwent a re-biopsy to verify the acquisition of resistance, as well as to the application of distinct first-line EGFR-TKI regimens although previous studies focusing on the rate of T790M acquisition after treatment with various EGFRTKIs have reported inconsistent results $(39,40)$. However, we observed that treatment with third-generation EGFRTKIs resulted in similar PFS in the three PD-L1 expression groups, and the data were comparable to those of a previous clinical trial of 2 nd-line osimertinib (9). These findings implicate that although PD-L1 expression may indicate the occurrence of de novo resistance to EGFR pathway inhibitors, the T790M mutation may still be acquired, and in that case $E G F R$ pathway inhibition may still be effective.

A possible mechanism of de novo resistance to EGFRTKI in patients with PD-L1 high lung adenocarcinoma with $E G F R$ mutation has been previously suggested, involving $M E T$ amplification and $K R A S$ mutations (30). In this study we showed that PD-L1 high EGFR mutant samples were significantly enriched with JAK-STAT pathway and MUC16 mutation using NCCRI and TCGA datasets. The JAKSTAT pathway mediates cytokine signaling and involved 
in promoting cell proliferations $(41,42)$. While JAK-STAT pathway upregulates PD-L1 expression $(43,44)$, JAK-STAT pathway was also shown to be associated with resistance to EGFR mutant lung cancer in a preclinical study (28). In addition, MUC16 mutation is associated with JAK-STAT3 pathway in tumorigenesis and metastasis (29). Altogether, these findings suggest that PD-L1 expression may indicate the activation of JAK-STAT pathway which can act as a de novo resistance mechanism to EGFR-TKI.

Combinations of EGFR-TKIs and anti-PD-1 antibodies to treat PD-L1-high, EGFR-mutated lung adenocarcinoma are being currently tested by clinical trials. However, treatment failure has frequently been reported due to the elevated incidence of pulmonary and hepatic toxicity (45). In light of our findings, combination therapies based on the inhibition of both EGFR and JAK-STAT pathways may prove effective for selected patients with PD-L1high, EGFR-mutated lung adenocarcinoma, especially those developing primary resistance to first-line EGFRTKI therapy. Preclinical studies have already demonstrated that JAK2 inhibition sensitizes resistant EGFR mutant lung adenocarcinoma to EGFR-TKI (46). A phase 1b clinical trial demonstrated that the combination of ruxolitinib, a JAK inhibitor, and afatinib is well tolerated but show modest therapeutic efficacy, with an overall response rate of $23.3 \%$ (47). Another clinical trial is ongoing to evaluate the efficacy of the combination of itacitinib, a JAK1 inhibitor, and osimertinib (ClinicalTrials.gov Identifier: NCT02917993). Should the efficacy of these combination therapies be confirmed, our study may pave the way for the use of PD-L1 expression as a new biomarker to assess the effect of these treatments.

\section{Conclusions}

This study has some limitations mostly derived from a retrospective analysis. However, our findings were consistent with previous studies and supported by genomic and transcriptomic analysis of open-source databases. The antibodies to test PD-L1 expression were not identical throughout patients, although the two assays used in this study (22C3 and SP263) had been reported to have fair concordance rates (48).

In conclusion, we demonstrated that PD-L1 strong expression predicts poor response to first-or secondgeneration EGFR-TKI in treatment naïve advanced NSCLC, and that PD-L1 expression may indicate underlying de novo resistance mechanism including JAK-
STAT pathway. To illustrate the association of PD-L1 expression and resistance to EGFR-TKI more in depth, comprehensive genomic and transcriptomic analyses on these tumors along with annotated clinical features and treatment outcome data are warranted.

\section{Acknowledgments}

We would like to thank Editage (www.editage.co.kr) for English language editing. This manuscript was partly presented at the IASLC 2019 World Conference on Lung Cancer (WCLC); Barcelona, Spain, September 7 - 10, 2019 in a poster presentation.

Funding: This study was funded by a grant from the SNUBH Research Fund (grant number: 13-2017-002 to SHK).

\section{Footnote}

Reporting Checklist: The authors have completed the REMARK reporting checklist. Available at http://dx.doi. org/10.21037/tlcr-20-893

Data Sharing Statement: Available at http://dx.doi. org/10.21037/tlcr-20-893

Peer Review File: Available at http://dx.doi.org/10.21037/ tlcr-20-893

Conflicts of Interest: All authors have completed the ICMJE uniform disclosure form (available at http://dx.doi. org/10.21037/tlcr-20-893). Dr. TMK reports grants from AZ-KHIDI, outside the submitted work; Dr. DWK reports research funding to institution: Alpha Biopharma, Amgen, Astrazeneca/Medimmune, Boehringer-Ingelheim, DaiichiSankyo, Hanmi, Janssen, Merus, Mirati Therapeutics, MSD, Novartis, ONO Pharmaceutical, Pfizer, Roche/ Genentech, Takeda, TP Therapeutics, Xcovery, and Yuhan and travel and accommodation support for advisory board meeting attendance: Amgen, Daiichi-Sankyo. The other authors have no conflicts of interest to declare.

Ethical Statement: The authors are accountable for all aspects of the work in ensuring that questions related to the accuracy or integrity of any part of the work are appropriately investigated and resolved. This study was performed in accordance with the Declaration of Helsinki (as revised in 2013). This study was approved by the 
Institutional Review Board of Seoul National University Bundang Hospital (IRB No. B-1808/487-101). Consent to participate was waived by the ethics board.

Open Access Statement: This is an Open Access article distributed in accordance with the Creative Commons Attribution-NonCommercial-NoDerivs 4.0 International License (CC BY-NC-ND 4.0), which permits the noncommercial replication and distribution of the article with the strict proviso that no changes or edits are made and the original work is properly cited (including links to both the formal publication through the relevant DOI and the license). See: https://creativecommons.org/licenses/by-nc-nd/4.0/.

\section{References}

1. Hanna N, Johnson D, Temin S, et al. Systemic Therapy for Stage IV Non-Small-Cell Lung Cancer: American Society of Clinical Oncology Clinical Practice Guideline Update. J Clin Oncol 2017;35:3484-515.

2. NCCN Clinical Practice Guidelines in Oncology NonSmall Cell Lung Cancer Version 1. 2021 - December 03, 2020 [Internet]. nccn.org; Available online: https://www. nccn.org/professionals/physician_gls/pdf/nscl.pdf

3. Planchard D, Popat S, Kerr K, et al. Metastatic non-small cell lung cancer: ESMO Clinical Practice Guidelines for diagnosis, treatment and follow-up. Ann Oncol 2018;29:iv192-iv237.

4. Wu YL, Planchard D, Lu S, et al. Pan-Asian adapted Clinical Practice Guidelines for the management of patients with metastatic non-small-cell lung cancer: a CSCO-ESMO initiative endorsed by JSMO, KSMO, MOS, SSO and TOS. Ann Oncol 2019;30:171-210.

5. Zhou C, Di Yao L. Strategies to Improve Outcomes of Patients with EGRF-Mutant Non-Small Cell Lung Cancer: Review of the Literature. J Thorac Oncol 2016;11:174-86.

6. Yu HA, Arcila ME, Rekhtman N, et al. Analysis of tumor specimens at the time of acquired resistance to EGFR-TKI therapy in 155 patients with EGFR-mutant lung cancers. Clin Cancer Res 2013;19:2240-7.

7. Katakami N, Atagi S, Goto K, et al. LUX-Lung 4: a phase II trial of afatinib in patients with advanced nonsmall-cell lung cancer who progressed during prior treatment with erlotinib, gefitinib, or both. J Clin Oncol 2013;31:3335-41.

8. Miller VA, Hirsh V, Cadranel J, et al. Afatinib versus placebo for patients with advanced, metastatic non- small-cell lung cancer after failure of erlotinib, gefitinib, or both, and one or two lines of chemotherapy (LUXLung 1): a phase $2 \mathrm{~b} / 3$ randomised trial. Lancet Oncol 2012;13:528-38.

9. Mok TS, Wu YL, Ahn MJ, et al. Osimertinib or platinumpemetrexed in EGFR T790M-positive lung cancer. N Engl J Med 2017;376:629-40.

10. Blank C, Gajewski TF, Mackensen A. Interaction of PD$\mathrm{L} 1$ on tumor cells with PD-1 on tumor-specific T cells as a mechanism of immune evasion: implications for tumor immunotherapy. Cancer Immunol Immunother 2005;54:307-14.

11. Shin J, Chung JH, Kim SH, et al. Effect of platinum-based chemotherapy on PD-L1 expression on tumor cells in nonsmall cell lung cancer. Cancer Res Treat 2019;51:1086-97.

12. Mu CY, Huang JA, Chen Y, et al. High expression of PD-L1 in lung cancer may contribute to poor prognosis and tumor cells immune escape through suppressing tumor infiltrating dendritic cells maturation. Med Oncol 2011;28:682-8.

13. Dal Bello MG, Alama A, Coco S, et al. Understanding the checkpoint blockade in lung cancer immunotherapy. Drug Discov Today 2017;22:1266-73.

14. Aguiar Jr PN, De Mello RA, Hall P, et al. PD-L1 expression as a predictive biomarker in advanced non-small-cell lung cancer: updated survival data. Immunotherapy 2017;9:499-506.

15. Bonanno L, Schiavon M, Nardo G, et al. Prognostic and predictive implications of EGFR mutations, EGFR copy number and KRAS mutations in advanced stage lung adenocarcinoma. Anticancer Res 2010;30:5121-8.

16. Han HS, Lim SN, An JY, et al. Detection of EGFR mutation status in lung adenocarcinoma specimens with different proportions of tumor cells using two methods of differential sensitivity. J Thorac Oncol 2012;7:355-64.

17. Jeon SH, Kim HW, Kim BN, et al. Comparison of PNA clamping-assisted fluorescence melting curve analysis and PNA clamping in detecting EGFR mutations in matched tumor tissue, cell block, pleural effusion and blood of lung cancer patients with malignant pleural effusion. In Vivo 2019;33:595-603.

18. Won JK, Keam B, Koh J, et al. Concomitant ALK translocation and EGFR mutation in lung cancer: a comparison of direct sequencing and sensitive assays and the impact on responsiveness to tyrosine kinase inhibitor. Ann Oncol 2015;26:348-54.

19. Okayama H, Kohno T, Ishii Y, et al. Identification of genes upregulated in ALK-positive and EGFR/KRAS/ 
ALK-negative lung adenocarcinomas. Cancer Res 2012;72:100-11.

20. Collisson E, Campbell J, Brooks A, et al. Comprehensive molecular profiling of lung adenocarcinoma. Nature 2014;511:543-50.

21. Cerami E, Gao J, Dogrusoz U, et al. The cBio cancer genomics portal: an open platform for exploring multidimensional cancer genomics data. Cancer Discov 2012;2:401-4.

22. Eisen MB, Spellman PT, Brown PO, et al. Cluster analysis and display of genome-wide expression patterns. Proc Natl Acad Sci U S A 1998;95:14863-8.

23. Skov BG, Rørvig SB, Jensen THL, et al. The prevalence of programmed death ligand-1 (PD-L1) expression in non-small cell lung cancer in an unselected, consecutive population. Mod Pathol 2020;33:109-17.

24. Mootha VK, Lindgren CM, Eriksson KF, et al. PGC-1 $\alpha$ responsive genes involved in oxidative phosphorylation are coordinately downregulated in human diabetes. Nat Genet 2003;34:267-73.

25. Subramanian A, Tamayo P, Mootha VK, et al. Gene set enrichment analysis: a knowledge-based approach for interpreting genome-wide expression profiles. Proc Natl Acad Sci U S A 2005;102:15545-50.

26. Liberzon A, Birger C, Thorvaldsdóttir H, et al. The Molecular Signatures Database (MSigDB) hallmark gene set collection. Cell Syst 2015;1:417-25.

27. Harada D, Takigawa N, Kiura K. The role of STAT3 in non-small cell lung cancer. Cancers 2014;6:708-22.

28. Kim SM, Kwon OJ, Hong YK, et al. Activation of IL6R/JAK1/STAT3 signaling induces de novo resistance to irreversible EGFR inhibitors in non-small cell lung cancer with T790M resistance mutation. Mol Cancer Ther 2012;11:2254-64.

29. Lakshmanan I, Salfity S, Seshacharyulu P, et al. MUC16 regulates TSPYL5 for lung cancer cell growth and chemoresistance by suppressing p53. Clin Cancer Res 2017;23:3906-17.

30. Su S, Dong ZY, Xie Z, et al. Strong Programmed Death Ligand 1 Expression Predicts Poor Response and De Novo Resistance to EGFR Tyrosine Kinase Inhibitors Among NSCLC Patients With EGFR Mutation. J Thorac Oncol 2018;13:1668-75.

31. Hsu KH, Huang YH, Tseng JS, et al. High PD-L1 expression correlates with primary resistance to EGFRTKIs in treatment naïve advanced EGFR-mutant lung adenocarcinoma patients. Lung Cancer 2019;127:37-43.

32. Yang CY, Liao WY, Ho CC, et al. Association between programmed death-ligand 1 expression, immune microenvironments, and clinical outcomes in epidermal growth factor receptor mutant lung adenocarcinoma patients treated with tyrosine kinase inhibitors. Eur J Cancer 2020;124:110-22.

33. Brown H, Vansteenkiste J, Nakagawa K, et al. Programmed cell death ligand 1 expression in untreated EGFR mutated advanced NSCLC and response to osimertinib versus comparator in FLAURA. J Thorac Oncol 2020;15:138-43.

34. Bai Y, Chen X, Hou L, et al. PD-L1 expression and its effect on clinical outcomes of EGFR-mutant NSCLC patients treated with EGFR-TKIs. Cancer Biol Med 2018;15:434-42.

35. Hastings $\mathrm{K}$, Yu H, Wei W, et al. EGFR mutation subtypes and response to immune checkpoint blockade treatment in non-small-cell lung cancer. Ann Oncol 2019;30:1311-20.

36. Lisberg A, Cummings A, Goldman J, et al. A phase II study of pembrolizumab in EGFR-mutant, PD-L1+, tyrosine kinase inhibitor naïve patients with advanced NSCLC. J Thorac Oncol 2018;13:1138-45.

37. Socinski MA, Jotte RM, Cappuzzo F, et al. Atezolizumab for first-line treatment of metastatic nonsquamous NSCLC. N Engl J Med 2018;378:2288-301.

38. West H, McCleod M, Hussein M, et al. Atezolizumab in combination with carboplatin plus nab-paclitaxel chemotherapy compared with chemotherapy alone as firstline treatment for metastatic non-squamous non-smallcell lung cancer (IMpower130): a multicentre, randomised, open-label, phase 3 trial. Lancet Oncol 2019;20:924-37.

39. Lee K, Kim Y, Jung HA, et al. Repeat biopsy procedures and $T 790 M$ rates after afatinib, gefitinib, or erlotinib therapy in patients with lung cancer. Lung Cancer 2019;130:87-92.

40. Yoon BW, Kim JH, Lee SH, et al. Comparison of T790M Acquisition Between Patients Treated with Afatinib and Gefitinib as First-Line Therapy: Retrospective Propensity Score Matching Analysis. Transl Oncol 2019;12:852-8.

41. Dutta P, Sabri N, Li J, et al. Role of STAT3 in lung cancer. JAKSTAT 2015;3:e999503.

42. Thomas SJ, Snowden JA, Zeidler MP, et al. The role of JAK/STAT signalling in the pathogenesis, prognosis and treatment of solid tumours. Br J Cancer 2015;113:365-71.

43. Doi T, Ishikawa T, Okayama T, et al. The JAK/STAT pathway is involved in the upregulation of PD-L1 expression in pancreatic cancer cell lines. Oncol Rep 2017;37:1545-54.

44. Mimura K, Teh JL, Okayama H, et al. PD-L1 expression is mainly regulated by interferon gamma associated 
with JAK-STAT pathway in gastric cancer. Cancer Sci 2018;109:43-53.

45. Liang H, Liu X, Wang M. Immunotherapy combined with epidermal growth factor receptor-tyrosine kinase inhibitors in non-small-cell lung cancer treatment. Onco Targets Ther 2018;11:6189-96.

46. Gao SP, Chang Q, Mao N, et al. JAK2 inhibition sensitizes resistant EGFR-mutant lung adenocarcinoma to tyrosine kinase inhibitors. Sci Signal 2016;9:ra33.

Cite this article as: Kang M, Park C, Kim SH, Yoon SW, Suh KJ, Kim YJ, Ock CY, Kim M, Keam B, Kim TM, Kim DW, Heo DS, Lee JS. Programmed death-ligand 1 expression level as a predictor of EGFR tyrosine kinase inhibitor efficacy in lung adenocarcinoma. Transl Lung Cancer Res 2021;10(2):699-711. doi: $10.21037 /$ tlcr-20-893
47. Park JS, Hong MH, Chun YJ, et al. A Phase Ib Study of the Combination of afatinib and ruxolitinib in EGFR mutant NSCLC with progression on EGFR-TKIs. Lung Cancer 2019;134:46-51.

48. Hirsch FR, McElhinny A, Stanforth D, et al. PD-L1 immunohistochemistry assays for lung cancer: results from phase 1 of the blueprint PD-L1 IHC assay comparison project. J Thorac Oncol 2017;12:208-22. 


\section{Supplementary}

Table S1 Comparison of T790M mutation between Exon 19 del and Exon 21 L858R

\begin{tabular}{lccc}
\hline T790M mutation detected from rebiopsy & Exon 19 del & Exon 21 L858R & P value \\
\hline No & 11 & 14 & 0.569 \\
Yes & 21 & 20 & \\
\hline
\end{tabular}

Table S2 Comparison of T790M mutation between first-line EGFR-TKIs applied

\begin{tabular}{|c|c|c|c|c|}
\hline T790M mutation detected from rebiopsy & Gefitinib & Erlotinib & Afatinib & $P$ value \\
\hline Yes & 17 & 22 & 2 & \\
\hline
\end{tabular}

EGFR, epidermal growth factor receptor; TKI, tyrosine kinase inhibitor.

Table S3 Significantly enriched pathways in PD-L1-high samples of the NCCRI dataset

\begin{tabular}{|c|c|c|c|}
\hline Pathway name & NES & NOM p-val & FDR q-val \\
\hline HALLMARK_ALLOGRAFT_REJECTION & 1.966872 & 0.002053 & 0.033883 \\
\hline HALLMARK_MITOTIC_SPINDLE & 1.958839 & 0.002101 & 0.024289 \\
\hline HALLMARK_INTERFERON_GAMMA_RESPONSE & 1.851237 & 0.016227 & 0.044286 \\
\hline HALLMARK_PI3K_AKT_MTOR_SIGNALING & 1.784433 & 0.014433 & 0.051453 \\
\hline HALLMARK_REACTIVE_OXYGEN_SPECIES_PATHWAY & 1.73737 & 0.024194 & 0.063726 \\
\hline HALLMARK_IL6_JAK_STAT3_SIGNALING & 1.729845 & 0.010142 & 0.057661 \\
\hline HALLMARK_IL2_STAT5_SIGNALING & 1.52697 & 0.032 & 0.110468 \\
\hline HALLMARK_APICAL_SURFACE & 1.519022 & 0.027833 & 0.100429 \\
\hline HALLMARK_COAGULATION & 1.37665 & 0.04878 & 0.16847 \\
\hline
\end{tabular}

NES, normalized enrichment score; NOM p-val, nominal p-value; FDR q-val, false discovery rate q-value. 
Table S4 Significantly enriched pathways in PD-L1-high samples of the TCGA dataset

\begin{tabular}{|c|c|c|c|}
\hline Pathway Name & NES & NOM p-val & FDR q-val \\
\hline HALLMARK_INTERFERON_GAMMA_RESPONSE & 1.713135 & 0.012121 & 0.20482 \\
\hline HALLMARK_IL6_JAK_STAT3_SIGNALING & 1.687761 & 0.010395 & 0.189793 \\
\hline HALLMARK_IL2_STAT5_SIGNALING & 1.549698 & 0.020704 & 0.208801 \\
\hline HALLMARK_INFLAMMATORY_RESPONSE & 1.544507 & 0.014644 & 0.193252 \\
\hline HALLMARK_APOPTOSIS & 1.544013 & 0.049383 & 0.175785 \\
\hline
\end{tabular}

NES, normalized enrichment score; NOM p-val, nominal p-value; FDR q-val, false discovery rate q-value. 
Table S5 Comparison of mutation profiles between PD-L1-high and PD-L1-low samples of the TCGA dataset

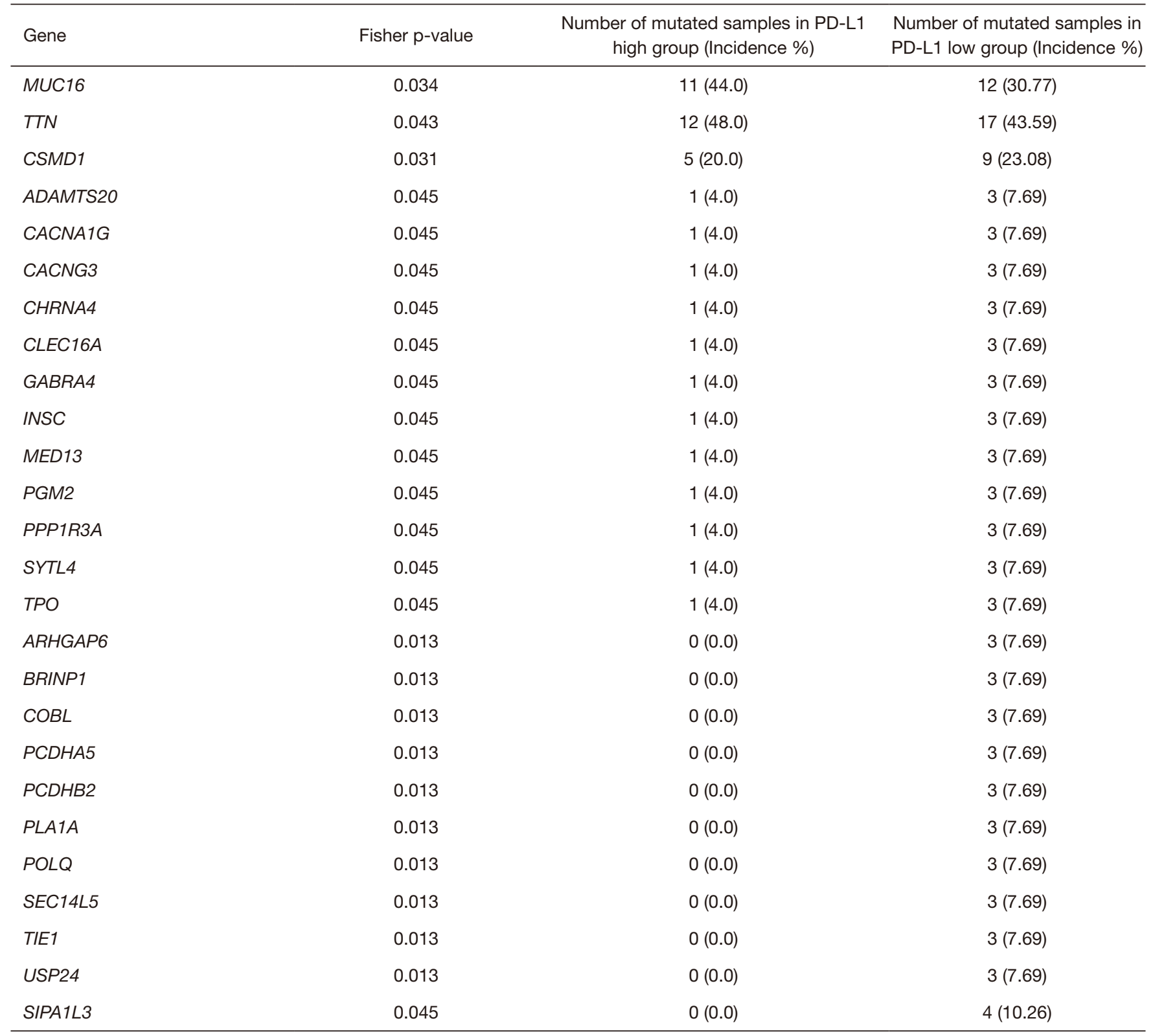

Only genes with significant differences are shown. 


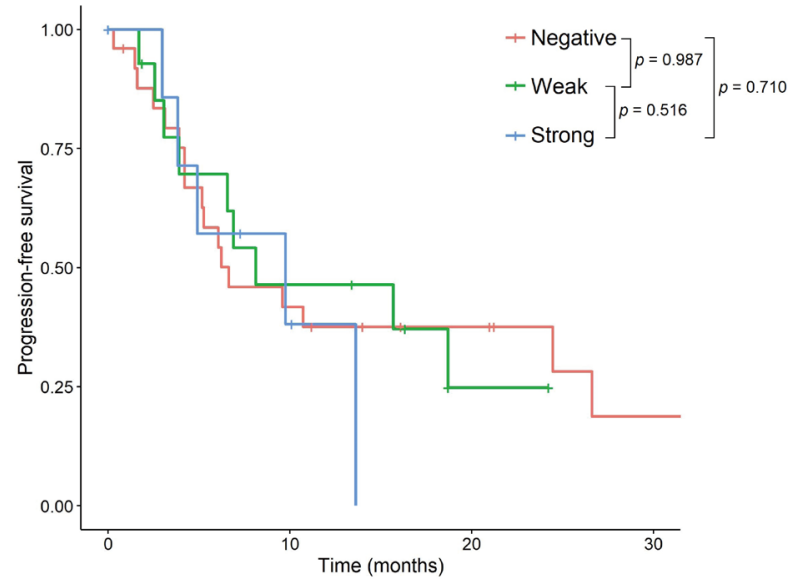

Figure S1 Kaplan-Meier curve of progression-free survival in patients receiving third-generation EGFR-TKI therapy. EGFR, epidermal growth factor receptor; TKI, tyrosine kinase inhibitor.

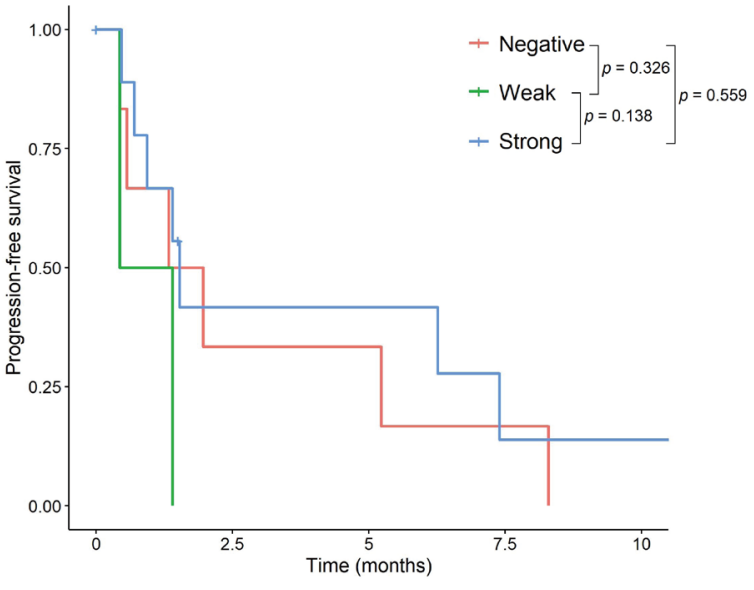

Figure S2 Kaplan-Meier curve of progression-free survival in patients receiving immune checkpoint inhibitor therapy. 